\title{
Physician Wellness Interventions and Distress Assessment During the Coronavirus Pandemic
}

\author{
Kimberly M. Robertello, $\mathrm{PhD}^{{ }^{*}}$, Karen M. Dahl, MD', Raed Khoury, MA, MPH' ${ }^{1}$ David Christensen, MD'
}

\begin{abstract}
Introduction: Physician burnout and interventions to decrease physician burnout on organizational and individual levels have been theorized and implemented. The Coronavirus pandemic has increased the need to continue physician burnout assessment and increase wellness programming. Few studies have had the opportunity to address physician wellness amidst the Coronavirus pandemic using the strategy of multi-dimensional interventions. The purpose of this study is to document interventions to reduce burnout and stress among physicians during the Coronavirus pandemic while assessing overall physician distress and well-being.

Methods: A cohort study of 304 pediatric physicians at a large healthcare organization in the United States was designed to measure distress in physicians using the Well-Being Index. Participation was voluntary. A third-party collected and disseminated de-identified aggregate data through a secure website portal. Organizational and individual wellness initiatives were introduced to the cohort population and voluntary participation tracked.

Results: 145 (48\%) Well-Being Index assessments were completed between March 16 and September 30, 2020. Mean distress over the 6-month period was 1.22, with high distress indicated by a score greater than 3.0. Monthly averages show a $111.5 \%$ decrease in distress scores during the time period. Over $91 \%$ of respondents reported feeling "somewhat" or "very supported" by the organization, and these respondents had overall low mean distress scores.

Conclusion: Overall, the cohort population experienced decreased distress levels at program initiation and during the observation period compared to national physician distress data. Contributing factors may be participation in various organizational and individual interventions to target well-being, perceived support from the organization, or regional implications of the Coronavirus pandemic on population health.
\end{abstract}

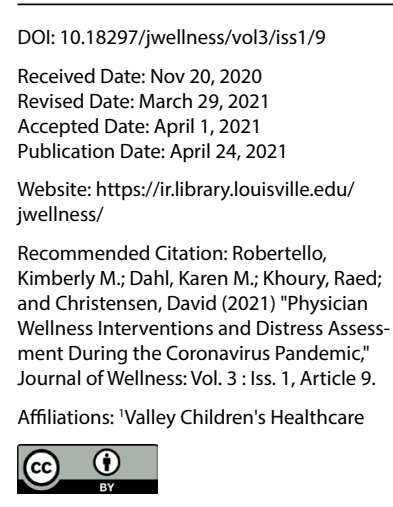

DOI: 10.18297/jwellness/vol3/iss1/9

20,2020

Website: https://ir.library.louisville.edu/ jwellness/

Recommended Citation: Robertello, Kimberly M.; Dahl, Karen M.; Khoury, Raed; and Christensen, David (2021) "Physician (2) Journal of Wellness: Vol. 3 : Iss. 1, Article 9.
J Affiliations: 'Valley Children's Healthcare

\section{INTRODUCTION}

Physician burnout is a professional syndrome which has been defined and widely studied [1-3]. Burnout syndrome is defined as a relationship between three measures: feelings of emotional exhaustion, increased cynicism related to one's work or an overall dehumanized approach, and a decreasing or negative self-worth [4]. Further research has addressed philosophical variance between individual and organizational approaches to address burnout, although little scientific study has been done to assess specific interventions and their results on physician well-being. Consensus among researchers is individual approaches to improving wellness and decreasing burnout are not sufficient without proper organizational support and endeavors [3-5]. Basic physical and mental health needs should be addressed first before higher-order organizational approaches [6]. There is also a burden of responsibility for individuals to implement personal factors to protect against burnout in ways which meet their unique needs.

Forty-eight percent (48\%) of physicians report coping with burnout most frequently using exercise. Eating junk food (35\%), alcohol use (26\%), and binge eating (21\%) are also reported coping mechanisms, which accentuate the need for encouraging positive preventative wellness models to address burnout [7]. Emphasizing mindfulness, gratitude, and resiliency can be positive preventative measures in reducing stressors during the Coronavirus pandemic [8]. These strategies may form the basis of preventative wellness for individuals who are not in an active state of depersonalization, cynicism, or low self-efficacy.

${ }^{*}$ Correspondence To: Kimberly Robertello

Email:krobertello@valleychildrens.org
While the benefits of comprehensive personal wellness are recognized, it cannot be inferred that the sole responsibility for physician wellness lies with the individual. In reality, organizational burden may be too cumbersome for any individual physician to overcome, and distress may become a significant inhibitor to solving issues [1]. Organizational support themes like flexibility, work-life integration, and self-care are impactful if implemented and practiced in a top-down approach. Organizational strategies (including acknowledging the problem, developing appropriate and targeted interventions, cultivating community, strengthening workplace culture, and promoting flexibility, resiliency, and work-life integration) are essential for success [5,9]. Educating staff members to recognize signs of distress such as anxiety, depression, substance abuse, and posttraumatic stress disorder can shift organizations to advocate for safety and accessibility for confidential resources [8]. Behind these strategies there needs to be visibility, support, and advocacy at every level [5].

In the midst of assessing and developing a response to physician burnout, medical systems were further stressed by the Coronavirus pandemic. As the pandemic took shape across the country, physicians provided care for others while anticipating and managing unprecedented personal, physi$\mathrm{cal}$, and psychological strain [10-14]. Challenges individuals may have faced before the Coronavirus pandemic, whether it be health-related, socioeconomic, or emotional, carry more weight in this uncertain time [7]. Together, these issues build a multi-dimensional problem. Burnout must continue to be assessed and interventions measured within the new paradigm

Copyright: $\odot 2021$ The author(s). This is an open access article distributed under the terms of the Creative Commons Attribution 4.0 International License (CC BY 4.0), which permits unrestricted use, distribution, and reproduction in any medium, provided the original author and source are credited. 
of the Coronavirus pandemic $[15,16]$. Individual resources should be enhanced to provide physicians with proactive measures to reduce stress and build resiliency. Organizational issues, which are becoming increasingly difficult during the pandemic, must still be addressed and overcome. Integration of chief wellness officers or clinician well-being programs into Covid-19 command centers or other decision-making bodies to ensure clinician psychological safety is a top organizational recommendation. Continuation of wellness programming and its supplementation should be a focus as the Coronavirus pandemic continues [17]. Specific implications for physicians in terms of preventative care, stress and long-term mental health effects must continue to be monitored and assessed $[16,18,19]$. The purpose of this study is to document interventions to reduce burnout among physicians during the Coronavirus pandemic while assessing overall physician distress and well-being.

\section{METHODS}

\section{Comprehensive Wellness Program}

This study was completed at a general pediatric medical and surgical facility serving 10 counties spanning rural and suburban areas. The target population for this intervention was the main campus medical staff members, specifically 304 full-time physicians. The Institutional Review Board (IRB) reviewed the proposed intervention (HSC2340) and concluded the study did not require IRB approval.

Before the Coronavirus pandemic restricted events and programming, the medical staff wellness program was designed to introduce wellness concepts within six dimensions of health: occupational, intellectual, social, mental, spiritual, and physical. For each dimension, current organizational initiatives and activities were documented. Potential activities for each area were developed and presented to the Chief Medical Officer for approval prior to implementation. The program was facilitated for medical staff members by a Wellness Specialist in coordination with the Physician Leadership Consultant, Executive Director and the Vice President of Patient Safety, Quality and Medical Affairs.

Post-March 2020, initiatives and events were re-organized to meet current social distancing and safety needs, as well as the increased need for stress management, physician resiliency and improved wellness. The following interventions were introduced or modified: Physician Mentorship Program, Continuing Medical Education Activities including the Physician Wellness Series and organizational sessions, new physician welcome and follow-up sessions, Covid-19 resources, community service projects, an outpatient ambulatory clinic for staff and family members, an online wellness platform, and complimentary lunches.

\section{Physician Mentorship Program}

The Physician Mentorship Program was introduced in August 2020. The program is structured using evidence-based practices to engage incoming physicians with a physician mentor. Mentees were approached before their organization start date to voluntarily participate in the program and complete a questionnaire to initiate the match process. Mentors were solicited for participation in the program by wordof-mouth, inter-organizational advertising, and referral. Mentors completed a questionnaire which would be shared with mentees to assist in the match process. Mentees reviewed questionnaires of 3-5 potential mentors and were asked to rank their top three choices for matches. Mentor/mentees were purposefully matched outside of their specialty to foster inter-professional development across the organization. An introductory meeting occurred in August 2020 to introduce participants and review program expectations. Mentors and mentees are expected to meet monthly from September 2020 to May 2021. Topics are pre-selected and sent to participants by the first of each month.

\section{Leadership Training}

Leadership training was facilitated for physicians who volunteered as mentors within the Physician Mentorship Program during the 2020-2021 mentorship series. Mentors were given the opportunity to attend three 45 -minute virtual Continuing Medical Education sessions lead by the organization's Physician Leadership Consultant. Content was developed as an Accredited Continuing Medical Education/California Medical Association Recurring Scheduled Series and approved for credit of 0.75 hours per session. Topics included leadership development, function, and role in the hierarchy of the workplace.

\section{Mentee Development}

Mentees within the Physician Mentorship Program voluntarily participated in three virtual 45-minute sessions about the shared mentee experience. The purpose of the mentee sessions was to increase engagement outside the provider's specialty and encourage support for personal and professional obstacles. Topics included transition to the organization, cultural beliefs, and work-life balance, among others. Mentees shared the long and short-term goals which they are working to develop with their mentors and discussed methods for continued success.

\section{Physician Wellness Series}

The Physician Wellness Series was introduced in January 2019 as part of the Accredited Continuing Medical Education/ California Medical Association to offer wellness strategies, evidence-based practices for implementation, and improve the wellness culture. After March 2020, the series was revised to highlight specific wellness topics within the scope of the Coronavirus pandemic.

\section{Continuing Medical Education: Organizational}

A variety of organizational Continuing Medical Education activities were scheduled to specifically address the Coronavirus pandemic. The scope of these activities ranged from clinical perspectives, treatment options, Multi-system Inflammatory Syndrome in Children, mental health for providers, and physician wellness. Sessions were offered virtually and were typically one hour or less in length.

\section{New Physician Welcome and Follow-up Sessions}

Prior to Coronavirus restrictions, the organization began a formal process to welcome new full-time physicians at the organization's main campus in October 2019. Physicians were given a welcome gift and an informational packet including organizational resources and community information. Follow-up interviews were scheduled at 30-days, 3-months, 6-months, and 12-months with our Wellness specialist or Vice President of Patient Safety, Quality and Medical Affairs to review needs, concerns, positive and negative feedback about the organization, and answer relevant questions. Themes from these conversations were recorded, and issues were addressed.

\section{Covid-19 Resources}

Covid-19 wellness resources were made available via the organization's intranet to all employees and medical staff members in April 2020. The information was categorized as follows: emotional health and well-being, immediate crisis needs, self-care resources, mindfulness and relaxation, healthy eating, sleep, physical activity, support and encouragement, self-assessments, counseling and support services, and communication. The material was sourced from national, state, and regional organizations and services. 


\section{Community Service Projects}

To address spiritual health and the concept of giving back to our community during the Coronavirus pandemic, locally based medical staff and their family members age 5-65 years were able to voluntarily participate in an ongoing service project at a local food bank. Volunteers donated four hours of time on recurring Saturday mornings to box food items for community distribution. Sessions occurred every other month.

\section{Ambulatory Clinic Response}

An outpatient, ambulatory care center opened to provide primary and urgent care on-site to employees, physicians, and their families in April 2020. The clinic was recognized as an early need in the Coronavirus pandemic when many private practices were closed or had very limited appointments. Services include well and sick visits, immunizations, Covid-19 screening and testing, laboratory and imaging services, prescription refills, telemedicine, and the availability of a licensed social worker. The clinic is staffed by two medical assistants, one Registered Nurse, and two physicians. The clinic is not meant to replace the patient's primary care physician, but to offer a convenient option to receive care quickly [20].

\section{Online Wellness and Fitness Programming}

Three hundred four (304) physicians were given complimentary access to online wellness and fitness programming beginning on August 1, 2020. The content was accessible as live or pre-recorded sessions via the internet or mobile app. Physicians could view and participate in over 40 categories of wellness content individually, using the group class format, or by employing short classes at the beginning or end of department meetings. Classes range in length from one minute to over one hour. Each physician was eligible for four complimentary accounts to gift to friends or family members to encourage engagement. Both live classes with full audio and visual feedback and pre-recorded classes are available.

\section{Complimentary Lunches}

Physicians and other clinicians credentialed by the medical staff at the main campus location were given access to a complimentary lunch twice weekly beginning in April 2020. Individual, pre-packaged lunches were available at a consistent time in a secured location for pick-up on a first-come, firstserved basis. Lunches were purchased from local restaurants to help provide support for businesses during the Coronavirus pandemic.

\section{Well-Being Index}

Overall physician well-being was assessed using the Well-Being Index. The Well-Being Index purpose, function and design was introduced to physicians at the organization beginning March 1, 2020. The assessment tool was launched on March 16, 2020. An organizational link to register was sent to all physicians. The Well-Being Index is available through a secure internet site or via the mobile app download administered through MedEd Solutions. Participation was voluntary and anonymous. MedEd Solutions collected and disseminated de-identified aggregate data to designated organizational administrators through a secure website portal. Demographic information was collected by MedEd Solutions and stored confidentially for the purpose of creating a user profile [21]. During the registration process to access the Well-Being Index, physicians identified the frequency at which they wanted to perform ongoing re-assessments. Choices included one, three, and six month intervals. At the pre-selected interval, individuals were prompted to voluntarily re-assess using the email address provided during their initial registration. Re-assessment is promoted within the instrument due to results in previous studies linking physician use of the tool with positive behavioral intention to improve their personal well-being [21].

After registration, participants were asked a 9-question inventory to address specific dimensions of distress and well-being: likelihood of burnout, severe fatigue, suicidal ideation, quality of life, meaning in work, work-life integration, medical error, and overall well-being [22]. The Well-Being Index has been independently validated in previous published studies [23]. The survey and scoring scale are included in Appendix A. Items 1-7 are answered using a yes/no format with one point assigned for each "yes". Items 8-9 are scored by two variables: meaning-in-work and work-life integration. Positive meaning-in-work and work-life integration decreases the distress score by one point each. A neutral response for each is equal to zero points, and a reported low level of meaning-in-work and work-life integration equals an increase by one point each. The total score for the Well-Being Index ranges from -2.0 (lowest risk) to 9.0 (highest risk), with scores over 3.0 signifying distress. Two additional questions drafted by the organization were included with the Well-Being Index to assess perceived level of support during the Coronavirus pandemic and overall medical staff wellness support. These questions were scored using descriptive statistics and do not contribute to the overall distress score.

\section{RESULTS}

\section{Engagement}

Physician engagement was measured for each wellness initiative or program offered between March and September 2020. Participation in all programs were voluntary and were not incentivized. The number of possible participants varied due to physician population fluctuations as a result of new hires, intermittent leaves of absence, resignation, termination, or retirement. Engagement in each program is included in Table 1.

Table 1: Program Engagement

\begin{tabular}{l|ll}
\hline Name of Program/Session & \multicolumn{1}{l}{$n$} & Frequency \\
\hline $\begin{array}{l}\text { Physician Mentor Program } \\
\text { Continuing Medical Education }\end{array}$ & 40 & $\begin{array}{l}\text { Monthly } \\
\text { (one-on-one meetings; } \\
\text { quarterly group meetings) }\end{array}$ \\
$\begin{array}{l}\text { (Physician Wellness Series) } \\
\text { Continuing Medical Education }\end{array}$ & 67 & 2 sessions \\
New Physician Welcome and & 40 & 9 sessions \\
Follow-up Sessions & 18 & As needed \\
Community Service Projects & 14 & Bi-monthly \\
$\begin{array}{l}\text { Ambulatory Clinic Response (telemedicine) } \\
\text { Ambulatory Clinic Response (walk-in visits) }\end{array}$ & 844 & $\begin{array}{l}\text { As needed } \\
\text { Online Wellness and Fitness Programming }\end{array}$ \\
$\begin{array}{l}\text { Complimentary Healthy Lunches } \\
162\end{array}$ & 150 & As needed \\
\hline
\end{tabular}

\section{Well-Being Index Assessment}

Three-hundred four (304) physicians were invited to voluntarily participate in the Well-Being Index. Initial assessments and re-assessments varied by month and individual. In March 2020, 81 physicians completed their initial Well-Being Index assessment. A total of 145 initial assessments were completed between March 16 and September 30, 2020; 91 re-assessments occurred during the same time period. Re-assessments were voluntary and were completed at the physician's pre-selected time interval during initial registration. Re-assessment frequency varied from physician to physician. Figure 1 (next page) shows monthly distribution of assessments and re-assessments. 
Intial and Re-Assessments by Month

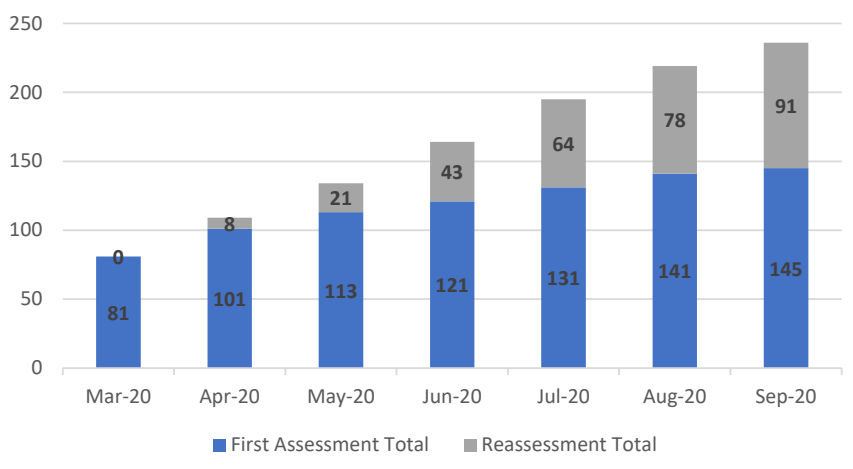

Figure 1: Monthly distribution of assessments and re-assessments

De-identified aggregate data was analyzed by overall distress score, and was further characterized by variables including gender, years since medical school graduation, and medical specialty. Any characterized score with a response rate less than $5(n<5)$ was not shared in the aggregate data from MedEd Solutions with the designated organizational administrators.

\section{Distress}

Physician distress was measured by overall well-being scores. Scores above 3.0 indicate distress. The mean cumulative physician distress score from the date of implementation through September 30, 2020 was $1.22(n=145)$. Monthly mean distress scores for initial and re-assessments are shown in Figure 2. From March to September 2020, overall distress for all respondents decreased by $111.5 \%$.

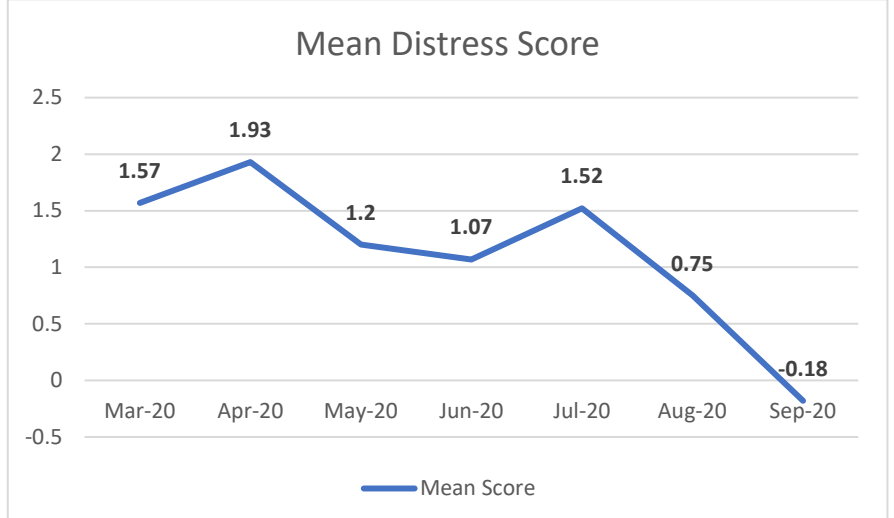

Figure 2: Monthly mean distress scores for initial and re-assessments
Percent Physicians with High Levels of Distress by Month

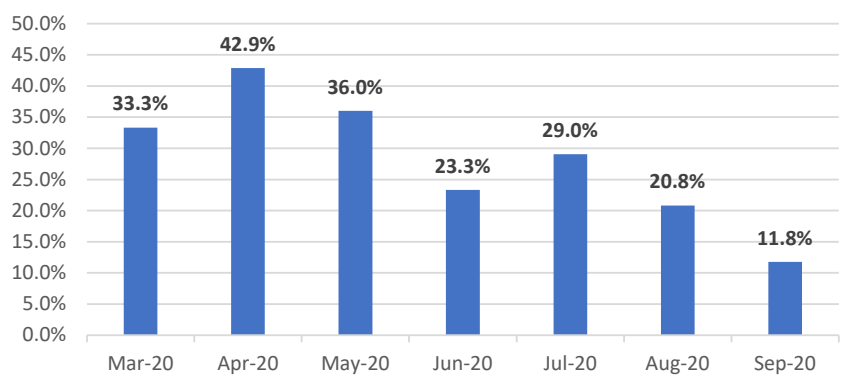

Figure 3: Monthly mean distress scores for initial and re-assessments

The mean percentage of physicians experiencing high distress during the intervention period was $30.08 \%(n=44)$. Over the 6-month intervention period, the percent of physicians within the organization experiencing high levels of distress, as indicated by a Physician Well-Being Index score of 3.0 or above, gradually declined (see Figure 3).

\section{Gender}

The distribution of respondents by gender is as follows: 72 female respondents (49.7\%), 69 male respondents $(47.6 \%)$, and 4 respondents who did not identify gender $(2.8 \%)$. Female physicians recorded higher Well-Being Index distress score means than their male counterparts. From March 16 through September 30, 2020, the mean cumulative female distress score was $1.39 \pm$ SD $2.48(n=72)$ and the mean cumulative male distress score was $1.09 \pm$ SD $2.33(n=69)$. Four participants did not identify gender, and mean distress scores of that subset population were not shared by MedEd solutions to protect anonymity.

\section{Number of Years Since Medical School Graduation}

Distress was analyzed by groupings of number of years since medical school graduation. Two (2) respondents were in the under 5-year category and their responses were not included by MedEd Solutions to protect anonymity. For each category, the percent of physicians in the organization with a Physician Well-Being Index score greater than 3.0 (indicating distress) was calculated. Mean distress scores, sample sizes, number, and percentages of physicians in each category with high distress scores are shown in Table 2. For each category, organizational scores by years' post-graduation were lower than the reported national physician distress scores by identical groupings of 14,900 US physicians [24].

Table 2: Details of physicians in each category with high distress scores

\begin{tabular}{|c|c|c|c|c|}
\hline $\begin{array}{l}\text { \# Years Since Medical } \\
\text { School graduation }\end{array}$ & $\begin{array}{l}\text { Sample } \\
\text { Size }(n)\end{array}$ & $\begin{array}{l}\text { Distress Score } \\
\text { Mean }\end{array}$ & $\begin{array}{l}\text { n Physicians with a High } \\
\text { Distress Score (>3.0) }\end{array}$ & $\begin{array}{l}\text { \% Physicians with a High } \\
\text { Distress Score (>3.0) }\end{array}$ \\
\hline Under 5 & 2 & NA & NA & NA \\
\hline 5-14 years & 61 & 1.31 & 17 & $28.09 \%$ \\
\hline $15-24$ years & 34 & 1.74 & 14 & $40.58 \%$ \\
\hline Over 25 years & 48 & 0.84 & 11 & $22.37 \%$ \\
\hline
\end{tabular}




\section{Medical Specialty}

During registration, physicians self-selected their specialty based on a custom list of specialties currently represented within the organization. Classifications include 28 specialties available including "other". Table 3 shows each specialty, number of respondents, and mean distress scores during the intervention period. The number of respondents of the following sub-specialties were less than 5 and were not shared by MedEd Solutions to protect anonymity: pediatric plastic surgery, pediatric cardiothoracic surgery, pediatric child advocacy, pediatric nephrology, pediatric physical medicine and rehabilitation, pediatric pathology, pediatric intensivists, pediatric rheumatology, pediatric urology, pediatric infectious disease, pediatric neurology/neurosurgery, pediatric pulmonology, pediatric radiology, and pediatric otolaryngology.

Table 3: Selected specialties by physicians

\begin{tabular}{l|lll} 
Specialty & Score & SD & $\begin{array}{l}\text { Sample } \\
\text { Size }\end{array}$ \\
\hline Pediatric Surgery & 1.0 & 2.11 & 5 \\
Pediatric Gastroenterology & -0.43 & 1.18 & 6 \\
Pediatric Hematology/Oncology & 0 & 2.15 & 7 \\
Pediatric Primary Care & 1.7 & 2.34 & 29 \\
Pediatric Endocrinology & -1.25 & 1.09 & 5 \\
Pediatric Orthopaedics & -0.29 & 1.28 & 5 \\
Pediatric Critical Care & 3.08 & 2.81 & 8 \\
Pediatric Hospitalists & 0.83 & 1.4 & 8 \\
Pediatric Maternal-Fetal & 0.25 & 2.77 & 5 \\
Pediatric Anesthesiology & 3.55 & 2.61 & 13 \\
Pediatric Neonatology & 0.42 & 1.19 & 7 \\
Pediatric Cardiology & 2.07 & 2.19 & 7 \\
Pediatric Emergency Medicine & 1.25 & 1.85 & 6 \\
Other & 0.58 & 1.93 & 10
\end{tabular}

\section{Coronavirus Response}

On July 15th, two questions were added to the Well-Being Index assessment tool that were organization-specific and not part of the original assessment as developed by the Mayo Clinic. The first question was "How supported have you felt as a medical staff member during the Covid-19 pandemic?" Responses range from "not supported at all", "less supported", "neutral", "somewhat supported", and "very supported". The second question asked, "Do you feel you have been provided meaningful wellness support/activities by [the organization]?" Available response choices were "yes", "somewhat", "no", or "I don't know".

Analysis of the first question (Covid-19 response) included number of respondents and their overall mean distress score if $n>5$. From July 15 to September 30, 2020, 30 respondents (66.67\%) felt "very supported", 11 (24.4\%) respondents felt "somewhat supported", 1 (2.2\%) respondent felt "neutral", and $3(6.67 \%)$ respondents felt "less supported". No respondents chose the option "not supported at all". Mean distress scores for respondents who reported "very supported" and

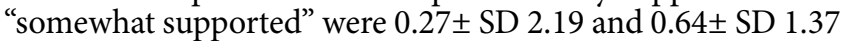
respectively. Mean distress scores for categories with less than 5 respondents were not reported.

Analysis of the second question, "[d]o you feel you have been provided meaningful wellness support/activities by [the organization]" had 45 responses from July 15 to September 30, 2020. Twenty-seven (60\%) respondents reported "yes", $12(26.67 \%)$ reported "somewhat", 4 (8.89\%) reported "no", and $2(4.44 \%)$ reported "I don't know". Mean distress scores of the respondents reporting "yes" were $0.16 \pm$ SD 2.06 and respondents reporting "somewhat" were $0.86 \pm$ SD 1.88. Mean distress scores for categories with less than 5 respondents were not reported.

\section{DISCUSSION}

The overall decrease in the organization's level of self-reported physician distress by $111.5 \%$ from March to September 2020 was remarkable in terms of the existing Coronavirus pandemic. Slight increases of mean distress scores from March to April (1.57 to 1.93) and again from June to July (1.07 to 1.52) occurred, but the overall decrease during a time of intense change, social, and professional burden is noteworthy. Increases coincide with regional imposed stay-at-home orders, school closures, and increases in Coronavirus cases. Additionally, the strain of implementing a new electronic medical record system on April 25, 2020 was an added organizational burden. Figure 4 illustrates how the monthly number of new Coronavirus cases in the county may have influenced the percentage of physicians experiencing high distress levels (as indicated by distress scores $>3.0$ ).

\section{New COVID-19 cases compared to \% of physicians with high distress}

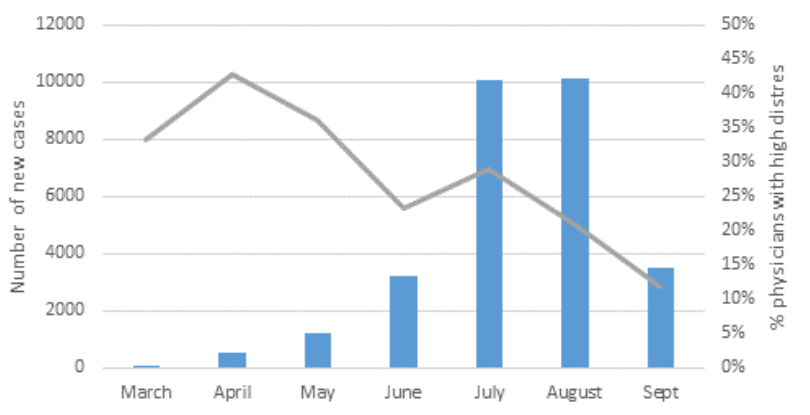

Figure 4: New coronavirus cases vs. high distress levels

Although the number of new cases was relatively low in March and April, all physicians were placed under unprecedented stressors from the Coronavirus pandemic including workplace modifications, adequate PPE availability concerns, risk of transmission, work-life integration, and societal impacts. Self-efficacy and increased organizational responses to the pandemic may have influenced later increases in new case numbers in July and August paired with contrasting decreasing percentages with physicians with high distress. It is noteworthy to relate this data to US and international studies of burnout and distress both at pre-pandemic levels and during the Coronavirus pandemic. Data from a recent study comparing pre- and post-pandemic data reveal $79 \%$ of physicians who have experienced burnout report the burnout beginning before the pandemic while $21 \%$ attribute burnout to post-pandemic. Workplace happiness has shifted post-pandemic, with decreases in happiness and increases in distress reported since last year [7]. A study of US and international physicians during the Coronavirus pandemic stated $64 \%$ of US physicians report self-perceived increased levels of burnout [21]. Data from this study indicated $30.08 \%$ of physicians had a high distress score $(>3.0)$ upon assessment over the 6-month period. Overall, in comparing reported distress data from the Well-Being Index as a single variant, this study's cohort population has shown a relatively low level of initial distress levels despite the onset of the Coronavirus pandemic.

The distress scores of the respondents by perceived level of organizational support during the Coronavirus pandemic was noteworthy. $66.7 \%$ of respondents felt "very supported" by the organization during the pandemic. The mean distress score for these respondents $(n=30)$ was $0.27 \pm S D 2.19$. Distress scores of those who felt "somewhat supported" (24.4\%) were slightly higher at $0.64 \pm$ SD 1.37 . The relationship between 
support and distress is something which should be explored in later studies. It cannot be assumed that a high perception of support correlates with lower distress scores. It would be of significance to determine if the physician's perception of support from the organization impacted their level of distress, especially during the pandemic. Likewise, perception of self-efficacy in response to the pandemic would be a similar variable of interest which may influence distress perception and assessment scores.

Although a small number of wellness activities were developed and implemented in second half of 2019, the institutional focus on wellness increased significantly during the Coronavirus pandemic. Pre-pandemic wellness activities were less frequent and varied in focus. Activities included social events for the medical staff and the introduction of weekly complimentary breakfasts and lunches beginning in November 2019. However, during the 6-month intervention period, $60 \%$ of respondents indicated support for wellness by the organization in contrast to $8.9 \%$ who indicated no wellness support. The "yes" group $(n=27)$ had a mean distress score of $0.16 \pm S D$ 2.06. Even those responding to the level of support as "somewhat" $(26.7 \%)$ had a low mean distress score of $0.86 \pm$ SD 1.88 . The data provokes the question of whether perceived support directly influences distress levels in physicians.

\section{LIMITATIONS}

This study is hindered by several factors. Wellness offerings did not have a clear start or end date, and no baseline data on wellness perception was gathered. Distribution of initial Well-Being Index assessments occurred on March 16, 2020, but it was purely coincidental in its relation to the Coronavirus pandemic and other wellness initiatives. However, the organizational wellness response increased due to the needs presented by the pandemic. Participation varied from person to person, as did the voluntary Well-Being Index assessments. Some may have taken part in one or two initiatives, where others may have had close to full participation. One may argue that those with lower distress scores had increased participation in wellness initiatives to increase well-being, and vice versa. If wellness is a completely internally motivated concept, one may question whether initiatives can affect distress. However, as seen in previous burnout studies with physician populations, there are many variables which can affect burnout that are outside of the physician's control. These organizational barriers to wellness cannot be overcome with self-assessments and a positive outlook alone [5]. Data from a 2021 report on physician wellness states $35 \%$ of respondents report their workplace offers a program to reduce stress and/ or burnout, yet $42 \%$ of respondents said they are "very unlikely" to participate in this program [7]. Part of this discrepancy is the perception of individual versus organizational accountability to address burnout, which leaves physicians less likely to participate in these efforts. The high level of perceived support with this cohort population paired with overall low distress scores may indicate a blossoming culture of wellness and organizational change. Promotion of resilience and self-care is just one of many components of a strong organizational wellness program [5]. An argument for the preventative value in wellness initiatives is possible and deserves further exploration.

Perceptions of time, ability, and desire to participate in various initiatives will vary from physician to physician. There was no specified administrative time set aside for physicians to engage in wellness activities. Physicians in this organization are members of one of seven different provider groups, and their time is not solely governed by medical staff demands and clinical care alone. Additionally, some may have felt greater hindrances resulting from impacts on their personal time which prohibited participation. Variables such as childcare and online school for children in the household, caring for sick or elderly family members, changes in schedules for other household members, and other societal changes could be significant. Traditionally, burdens of household responsibilities fall to women, despite their work status. The variance in work schedules during the pandemic for some specialties or departments may have a range of effects. Nationally, some specialties experienced a surge of patients, while physicians in other departments were idle or asked to work in unfamiliar settings. Positive organizational impacts during the pandemic at this organization included adequate PPE supplies and no change in their pre-Coronavirus pandemic salary and benefit levels, alleviating additional stresses.

Control group comparison is not always practical in a real-life work environment. Denying resources to physicians during a public health crisis was also unethical. It is arguable that the other medical staff members or hospital employees who did not have access to all of the interventions may have served as a control group. However, this concept does not account for the professional differences which may exist as they relate specifically to physician burnout, especially during the Coronavirus pandemic. Differences exist between clinical professions and burnout rates. Additionally, some interventions were available to non-physicians. Outside of the spectrum of a control group are influences of wellness interventions which have been present for some time at this organization or by the provider groups, for example Employee Assistance Programs and Critical Incident Debriefing. These types of programs are common in many organizations and will positively influence the wellness culture of an organization. The Well-Being Index assessment itself has limitations. Physicians have expressed the need to keep mental health information private. This may decrease the propensity to participate in a self-assessment of distress and well-being. Despite anonymity, some physicians have a general distrust of these types of assessments which lowers their engagement. A further area of study would be the propensity of distressed physicians to self-assess wellness and take corrective action. Furthermore, data analysis and inaccessibility of raw data was limited by MedEd Solutions to protect physician anonymity. Multi-variate analysis was not possible using this version of the Well-Being Index, nor is the ability to track individual distress data over time.

\section{CONCLUSION}

Overall, distress scores decreased during a time of medical, professional, and societal struggle in this organization. The Coronavirus pandemic, electronic medical record transition, and introductions to various wellness strategies all contributed to the lessons learned during this period. It will be valuable to continually evaluate distress data for physicians moving forward. Due to the voluntary nature of the Well-Being Index assessments and wellness programming participation, a causal relationship between wellness interventions and distress scores is unknown. However, the types of interventions offered, participation rates, and data reflecting organizational support are relevant information for others creating wellness programs in the future at similar organizations. The Coronavirus pandemic will continue for months to years. The long-lasting effects of the pandemic on the workforce are yet to be discovered.

\section{REFERENCES}

1. Maslach, C. Finding solutions to the problem of burnout. Counsult Psychol J Res Pract. 2017;69(2):143-152.

2. Schaufeli, W.B., M.P. Leiter, C. Maslach. Burnout: 35 years of research and practice. Career Dev. 2009;14(3):204-220.

3. Rozario, Duncan. Burnout, resilience and moral injury: How the wicked problems of health care defy solutions,

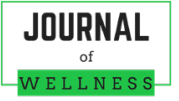


yet require innovative strategies in the modern era. Canadian Journal of Surgery. 2019;62(4):E6-E8.

4. Maslach, C., S.E. Jackson. The measurement of experienced burnout. Journal of Occupational Behaviour. 1981;2(2):99-113.

5. Shanafelt, Tait D, John H Noseworthy. Executive leadership and physician well-being: Nine organizational strategies to promote engagement and reduce burnout. Mayo Clinic Proceedings. 2017;92(1):129-146.

6. Shapiro DE, Duquette C, Abbott LM, Babineau T, Pearl A, Haidet P. Beyond burnout: A physician wellness hierarchy designed to prioritize interventions at the systems level. Am J Med. 2019.132(5), 556-563. https://doi. org/10.1016/j.amjmed.2018.11.028.

7. Kane L. 'Death by 1000 cuts': Medscape National physician burnout \& suicide report 2021. Medscape. January 22, 2021. Accessed March 5, 2021. https://www.medscape. com/slideshow/2021-lifestyle-burnout-6013456\# 1

8. Bansal, Priya, Theresa A Bingemann, Matthew Greenhawt, Giselle Mosnaim, Anil Nanda, John Oppenheimer, Hemant Sharma, David Stukus, Marcus Shaker. Clinician wellness during the COVID-19 pandemic: Extraordinary times and unusual challenges for the allergist/immunologist. The Journal of Allergy and Clinical Immunology. In Practice. 2020;8(6):1781-1790.e3.

9. Hofmeyer, Anne, Ruth Taylor, Kate Kennedy. Fostering compassion and reducing burnout: How can health system leaders respond in the Covid-19 pandemic and beyond? Nurse Education Today. 2020;94:104502. https:// www.ncbi.nlm.nih.gov/pmc/articles/PMC7295512/ https://doi.org/10/1016/j.net.2020.204502

10. Gardner, Rebekah L, Emily Cooper, Jacqueline Haskell, Daniel A Harris, Sara Poplau, Philip J Kroth, Mark Linzer. Physician stress and burnout: the impact of health information technology. Journal of the American Medical Informatics Association : JAMIA. 2019;26(2):106-114.

11. Ayanian JZ. Mental Health Needs of Health Care Workers Providing Frontline COVID-19 Care. JAMA Health Forum. [Internet]. April 1, 2020. Available from: https://jamanetwork.com/channels/health-forum/fullarticle/2764228doi:10.1001/ jamahealthforum.2020.0397

12. Greenberg, Neil, Mary Docherty, Sam Gnanapragasam, Simon Wessely. Managing mental health challenges faced by healthcare workers during covid-19 pandemic. BMJ (Clinical Research Ed.). 2020;368:m1211. . https://www. bmj.com/content/368/bmj.m1211/rapid-responses

13. Dutheil, Frédéric, Laurie Mondillon, Valentin Navel. PTSD as the second tsunami of the SARS-Cov-2 pandemic. Psychological Medicine. 2020;1-2:1-2.

14. Miguel-Puga, José Adán, Davis Cooper-Bribiesca, Francisco José Avelar-Garnica, Luis Alejandro Sanchez-Hurtado, Tania Colin-Martínez, Eliseo Espinosa-Poblano, Juan Carlos Anda-Garay, Jorge Iván González-Díaz, Oscar Bernardo Segura-Santos, Luz Cristina Vital-Arriaga, Kathrine Jáuregui-Renaud. Burnout, depersonalization, and anxiety contribute to post-traumatic stress in frontline health workers at COVID-19 patient care, a follow-up study. Brain and Behavior. 2021;11(3):e02007. . https://onlinelibrary.wiley.com/doi/ full/10.1002/brb3.2007

15. Sharifi M, Asadi-Pooya AA, Mousavi-Roknabadi RS. Burnout among healthcare providers of COVID-19; A systematic review of epidemiology and recommendations. Arch Acad Emerg Med. 2021. 9(1): e7. [Internet] 10 Dec 2020. Available from: https://www.ncbi.nlm.nih. gov/pmc/articles/PMC7812159/

16. Norful, Allison A, Adam Rosenfeld, Krista Schroeder, Jasmine L Travers, Sainfer Aliyu. Primary drivers and psychological manifestations of stress in frontline healthcare workforce during the initial COVID-19 outbreak in the United States. General Hospital Psychiatry. 2021;69:20-26; Epub ahead of print.

17. Dzau, Victor J, Darrell Kirch, Thomas Nasca. Preventing a parallel pandemic - A national strategy to protect clinicians' well-being. The New England Journal of Medicine. 2020;383(6):513-515. https://pubmed.ncbi.nlm.nih. gov/32402153/https://doi.org/10.1056/NEJMp2011027. Internet

18. Fiest, K.M., Parsons Leigh, J., Krewulak, K.D. et al. Experiences and management of physician psychological symptoms during infectious disease outbreaks: a rapid review. BMC Psychiatry 21, 91. [Internet]. 2021. Available from: https://bmcpsychiatry.biomedcentral.com/ articles/10.1186/s12888-021-03090-9\#citeas https://doi. org/10.1186/s12888-021-03090-9.

19. Moitra M, Rahman M, Collins PY, et al. Mental health consequences for healthcare workers during the COVID19 pandemic: A scoping review to draw lessons for LMICs. Front Psychiatry. 2021;12:602614. [Internet]. 2021 Jan 27. https://doi.org/10.3389/fpsyt.2021.602614.

20. Valley Children's Healthcare. [Internet]. California (CA): Valley Children's Family Care Center; [cited 2020 October 5]. Available from: https://george.internal.chccm.org/ depts/hr/empBenefits/Pages/ValleyChildrensFamilyCareCenter.aspx

21. Shanafelt, Tait D, Krista L Kaups, Heidi Nelson, Daniel V Satele, Jeff A Sloan, Michael R Oreskovich, Lotte N Dyrbye. An interactive individualized intervention to promote behavioral change to increase personal well-being in US surgeons. Annals of Surgery. 2014;259(1):82-88.

22. MedEd Web Solutions. [Internet]. Minnesota (MN): Well-Being Index. [cited 2020 October 1]. Available from https://www.mededwebs.com/well-being-index?hsCtaTracking $=\mathrm{c} 04 \mathrm{ec} 002-\mathrm{e} 27 \mathrm{f}-4443-90 \mathrm{~b} 3-4 \mathrm{f} 2 \mathrm{f} 6 \mathrm{caefb} 93 \% 7 \mathrm{C} 3$ 076efc4-34e5-4106-833d-2896dd55bf5a

23. Dyrbye, Liselotte N, Daniel Satele, Jeff Sloan, Tait D Shanafelt. Utility of a brief screening tool to identify physicians in distress. Journal of General Internal Medicine. 2013;28(3):421-427.

24. Rotenstein, Lisa S, Matthew Torre, Marco A Ramos, Rachael C Rosales, Constance Guille, Srijan Sen, Douglas A Mata. Prevalence of burnout among physicians: A systematic review. Journal of the American Medical Association. 2018;320(11):1131-1150. 


\section{Appendix A}

\section{Scoring}

Original items (1-7) are answered using a simple yes/no format. One point is assigned for each "yes"

\section{Table 1. Scoring of ePWBI}

Points assigned

1. Have you felt burned out from your work?

2. Have you worried that your work is hardening you emotionally?

3. Have you often been bothered by feeling down, depressed, or hopeless?

4. Have you fallen asleep while sitting inactive in a public place?

1

5. Have you felt that all the things you had to do were piling up so high that you could not overcome them?

1

6. Have you been bothered by emotional problems (such as feeling anxious, depressed, or irritable)?

7. Has your physical health interfered with your ability to do your daily work at home and/or away from home?

8. The work I do is meaningful to me

\begin{tabular}{|l|l|}
\hline $\begin{array}{l}\text { a low level of meaning in work } \\
\text { (response option of a 1 or 2) }\end{array}$ & +1 \\
\hline $\begin{array}{l}\text { a neutral level of meaning in work } \\
\text { (response option of } 3 \text { to } 5 \text { on the 7-point Likert scale) }\end{array}$ & 0 \\
\hline $\begin{array}{l}\text { A high level of meaning in work } \\
\text { (response option of a } 6 \text { or } 7 \text { on the 7-point Likert scale) }\end{array}$ & -1 \\
\hline 9. My work schedule leaves me enough time for my personal/family life & \\
\hline $\begin{array}{l}\text { lower satisfaction with work-life integration } \\
\text { (e.g. disagree; strongly disagree) }\end{array}$ & +1 \\
\hline Neutral & \\
\hline Higher satisfaction with work-life integration \\
(agree, strongly agree)
\end{tabular}

The total score for the eWBI ranges from -2 (lowest risk) to 9 (highest risk). 